\title{
Buchbesprechungen/Book Reviews
}

Albert von Rohr, Die Medizinische Poliklinik der Universität Zürich 1835 bis 1983. Georg Thieme Verlag, Stuttgart/New York 1983. 418 pp. 30 Abb. Die medizinische Poliklinik in Zürich entstand 1835, d.h. praktisch gleichzeitig mit der Universität und zu einer Zeit, in der erst sehr wenige solche Institute im deutschen Sprachraum existierten. Sie begann mit einem Hinterzimmer im Hinteramt, und obwohl sie sich seither dauernd vergröBert hat, leidet sie immer noch an Platzmangel. Dieser Überblick über die ersten 150 Jahre der Institution ist in $10 \mathrm{Kapitel}$ unterteilt, wovon jedes einem der 10 Direktoren dieses Zeitraums und seinen Aktivitäten geweiht ist. Die Geschichte der Poliklinik zerfällt in zwei große Abschnitte, jeder etwa die Hälfte der Zeit umfassend: erst die Armenklinik (Direktoren: H. Locher-Balber, Fr. Ernst, Fr. Goll, O. Wyss, H. Müller); dann das wissenschaftliche Abklärungs- und Forschungszentrum. Die Direktoren der ersten Periode waren respektable Praktiker, aber übten keine nennenswerte wissenschaftliche Forschung aus. Wie sollten sie auch bei ihrer Arbeitsüberlastung (Hausbesuche!) und der «Sparsamkeit» der Behörden! Die Wendung zur zweiten Periode war durch die Einführung der obligatorischen Krankenversicherung (1928) bedingt, die Armenärzte überflüssig machte. Sie erfolgte unter Nägeli und Löffler. Sie und ihre Nachfolger (Rossier, Hegglin, W.Siegenthaler) haben nicht nur als Praktiker, sondern gerade als Forscher internationalen Ruf genossen. Was sozialmedizinische Leistungen wie Reihendurchleuchtungen etc. nicht ausschloß!

All dies wird in durchaus leserlicher Form (manchmal vielleicht etwas zu ausführlich) erzählt und ist auch meist weit über die Lokalgeschichte hinaus interessant. Auch hier war z. B. die Poliklinik mit ihrem Frauen- und Kinderüberschuß die Mutter der Kinderheilkunde. Leider kein Index.

Erwin H. Ackerknecht

Liselotte Kuntner. Die Gebärhaltung der Frau; Schwangerschaft und Geburt aus geschichtlicher, völkerkundlicher und medizinischer Sicht. Hans Marseille, München 1984. 205 Seiten, 88 Abb.

Das zentrale Thema dieser Arbeit ist die Wiedereinführung des Gebärstuhls und der vertikalen Gebärhaltung der Frau, welche die Verfasserin als diplomierte Physiotherapeutin für die beste hält. Folgende Kapitel setzen das Buch zusammen: 
1. Geschichte der Frauenheilkunde, Geburtshilfe und Physiotherapie

2. Geburt und Geburtshilfe aus völkerkundlicher Sicht

3. Die Gebärhaltung der Frau

4. Die Geburtsvorbereitung

Im Mittelpunkt des ersten Kapitels stehen die indische und griechische Gynäkologie und Geburtshilfe und, mit Recht, Soranus. Und die Hebammen (Exzerpte von C. Müllers Arbeit über das Lötschental). Die Geschichte der Physiotherapie verdankt ihre Anwesenheit hier wohl der Auffassung der Autorin, daß Physiotherapie neben Chirurgie und Pharmakotherapie eine der drei großen Abteilungen der Therapie ist. Es ist natürlich gefährlich, Jahrtausende auf 30 Seiten zu komprimieren. Es entstehen dann solche Merkwürdigkeiten wie Aetius (6. Jahrhundert), Paulus (geb. 635), Savonarola (geb. 390), J. Rueff (geb. 1500) als «mittelalterliche» Ärzte, usw.

Das zweite Kapitel resümiert die Übersicht von C.S. Ford und neuere ethnomedizinische Forschungen von Schiefenhövel, Uhlig und G. Konrad.

Das dritte Kapitel gibt einen kurzen Überblick über 15 Gebärstellungen und ihre Verbreitung sowie Geschichte und Verbreitung des Gebärstuhls. Arbeiten über den Einfluß der Körperhaltung auf verschiedene Faktoren des Geburtsvorgangs wie intraabdominalen Druck, Hämodynamik, Lungenfunktion usw. werden referiert. Die Autorin empfiehlt hier eine von ihr als Physiotherapeutin entwickelte neue Preßtechnik (Exspiration mit Lippenbremse). Den Abschluß des Kapitels bilden ein Überblick über recht problematische Fragebogenantworten von 200 Frauen, die die Verfasserin wohl physiotherapiert oder geburtsvorbereitet bzw. -instruiert hatte. Die Hälfte sind Zweitgebärende, die das erste Mal horizontal, das zweite Mal vertikal gebaren und natürlich das zweite Mal begeistert waren (Zweitgebärende pflegen das zweite $\mathrm{Mal}$ auch horizontal sich viel wohler zu fühlen). Das vierte Kapitel referiert über die «Geburtsvorbereitung» von Read, Velvorsten, Lamaze usw., welche nach Frau Kuntners Meinung natürlich vor allem die krankengymnastische Atemtherapie enthalten muß. Und «Psychosomatik». (Ein paar Gebete taten einst mindestens dieselben Dienste als der heute modische Fremdwortsalat.) Das Buch ist reich und interessant illustriert. Es ist mit viel Fleiß geschrieben, aber die Zitate sind allzu häufig aus zweiter Hand. Und es ist viel propagandistischer und dogmatischer als etwa die von mir in Gesnerus 1983 (Heft 3-4) positiv besprochene Sondernummer von «Curare» über das gleiche Thema, inklusive des dortigen Artikels von Frau Kuntner. Das Buch sagt z. B. nichts über Nachteile der 
vertikalen Gebärstellung, die früheren Bettgeburten in Indien oder Griechenland, etc. Es ist sicher, daß an der heutigen Geburtshilfe noch manches zu ändern ist und wahrscheinlich auch an der Gebärhaltung, aber dazu müssen noch viel mehr und andere Daten vorliegen als nur - sicher interessante - historische und völkerkundliche. Oder sollen wir das totale Wickeln der Säuglinge, den automatischen Aderlaß, diverse widersinnige Wochenbettdiäten etc. etc. nur wieder einführen, weil sie auch jahrtausendealt und weitverbreitet waren?

Erwin H.Ackerknecht

Blas Bruni Celli, Bibliografía Hipocrática. Ediciones del Rectorado, Universidad Central de Venezuela, Caracas 1984. 507 pp., ill. Broschiert Bs. 180,00 .

Les bibliographies hippocratiques se suivent et ne se ressemblent pas. Il y a trois ans paraissait Cinq cents ans de bibliographie hippocratique 1473-1982 de Maloney et Savoie l'année passée nous a gratifiés avec Corpus Hippocraticum (et Corpus Galenicum) de Gerhard Fichtner à Tübingen, et voici Bibliografía Hipocrática de Blas Bruni Celli. C'est une œuvre originale et artisanale. Maloney et Fichtner avaient recours à l'électronique, Bruni a rédigé lui-même les fiches des 4496 citations de sa Bibliographie. La bibliographie de M. Bruni est le prolongement d'un catalogue de sa propre collection d'ouvrages d'auteurs médicaux de l'Antiquité classique. Son ambition était de créer une Bibliographie Hippocratique complète. En plus des volumes de sa collection privée l'auteur a dépouillé un certain nombre de monographies et de bibliographies courantes dont il donne la liste. Il a eu un coup de chance (ou de malchance?) quand parut la bibliographie de Maloney. Il se devait d'incorporer les citations de Maloney dans sa propre liste, et il reconnaît ouvertement que son ouvrage serait resté très imcomplet sans l'insertion de ses citations-là. On peut discuter s'il vaut mieux adopter l'ordre alphabétique pur et simple pour une bibliographie comme celle-ci, ou l'ordre chronologique, comme Maloney. La liste des sources bibliographiques comprend presque toutes bio-bibliographies, les catalogues et les traités usuels. On y trouve de la Bibliography of the History of Medicine le $\mathrm{N}^{\mathrm{o}}$ 10 (1970-1974), le $\mathrm{N}^{0} 15$ (1975-1979), mais le $\mathrm{N}^{\circ} 5$ (1964-1969) manque. Le fascicule $N^{0} 17$ (1981) est là, mais le fasc. 16 (1980) manque.

On n'en voudra pas trop au typographe vénézolan d'avoir écorché des noms d'auteurs et d'avoir malmené des titres, mais la lecture des épreuves a été faite avec une légèreté impardonnable. En parcourant rapidement l'index des noms d'auteurs on a relevé les coquilles suivantes: Gresemann 